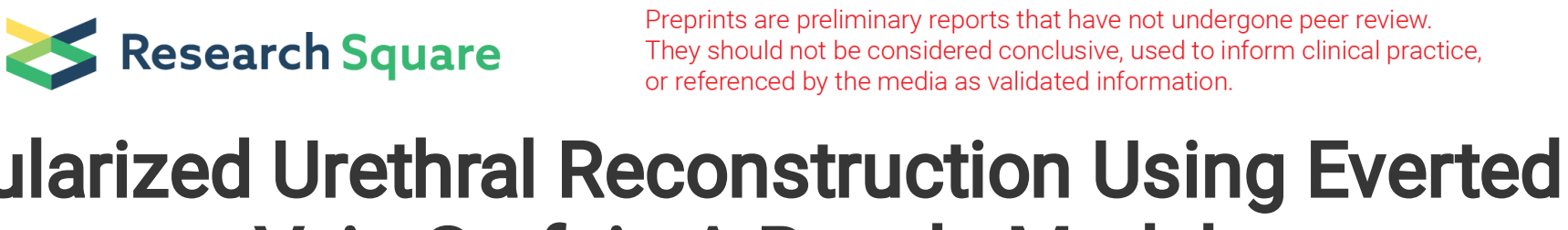 Saphenous Vein Graft in A Beagle Model
}

\section{Dan Li}

The First Affiliated Hospital of Wannan Medical College: Yijishan Hospital of Wannan Medical College

\section{Zhou Shen}

Anhui Provincial Hospital

\section{YujieXu ( $\sim$ xyj_1213@126.com)}

Yijishan Hospital of Wannan Medical College https://orcid.org/0000-0003-3820-2188

\section{Research article}

Keywords: Urethral stricture, hypospadias, Saphenous vein graft, Urethral reconstruction, Urethroplasty

Posted Date: October 6th, 2020

DOI: https://doi.org/10.21203/rs.3.rs-74278/v1

License: (9) This work is licensed under a Creative Commons Attribution 4.0 International License. Read Full License

Version of Record: A version of this preprint was published at BMC Urology on April 17th, 2021. See the published version at https://doi.org/10.1186/s12894-021-00833-4. 


\section{Abstract}

Background: The repair of anterior urethral stricture and hypospadias require urethral reconstruction. We conduct the study to investigate the efficacy of anterior urethral reconstruction using everted saphenous vein graft in tubular fashion.

Methods: Twelve male beagles were randomly divided into three groups: experimental $\operatorname{group}(n=5)$, control group $(n=5)$ and normal group $(n=2)$. A $3 \mathrm{~cm}$ defect in anterior urethra was created. Autologous saphenous vein graft was harvested. In experimental group, urethral defect was replaced by everted saphenous vein graft in tubular fashion. In control group, urethral reconstruction was performed using uneverted saphenous vein graft. At 6 months postoperatively, beagles in all groups received retrograde urethrography to evaluate urethral patency and were killed for histological examination.

Results: Retrograde urethrography showed the urethral caliber of beagles in experimental group were similar to those of normal. Ether urethral stricture or fistula were detected in all animals in control group. In experimental group, the everted saphenous vein graft completely integrated into the urethra. The reconstructed urethra had a wide lumen and was completely covered by urothelium. The subepithelial collagen and muscle fibers were well formed and highly organized. Everted saphenous vein graft had a high ability of neovascularization. In control group, the reconstructed segment had a fibrotic urethral lumen in which urothelium was not intact. And there were few newly formed capillaries.

Conclusions: Everted saphenous vein graft can be used as tubular substitute material for anterior urethral strictures and hypospadias.

\section{Background}

The urethra is a hollow elastic organ. Adult and pediatric disorders of the urethra including hypospadias, trauma, and stricture require surgical intervention to preserve urinary tract function. Repair of the anterior urethra is one of the most challenging problems in urology[1].

Autologous tissues have advantages of excellent biological compatibility, rapid and effective neovascularization. Oral mucosa and penile skin flap are preferred substituted materials for urethral reconstruction[2, 3]. Onlay urethroplasty and multi-stage repair are commonly used technique[4]. In some cases, these methods have problems including stricture recurrence, fistula formation, amount of donor material is not adequate. Harvesting oral mucosa is associated with morbidity at the donor site, such as submucosal scarring, pain, numbness, injury to salivary ducts[5, 6]. These issues highlight the critical need for the development of alternative material and reconstructive strategies for anterior urethral repair.

The saphenous vein is commonly used as vascular substitute material. It is easy to be harvested and almost without injury to its donor area. We previously performed urethroplasty in a rabbit model using everted saphenous vein as onlay graft and gained encouraging results. We published our hypothesis that everted vein graft could survive without graft necrosis and slough off[7]. Everted vein graft makes the 
endothelial cells outside to obtain blood supply, which may facilitate angiogenesis. Due to saphenous vein has a similar caliber as the urethra, we imagine using everted saphenous vein graft to repair anterior urethral defect in tubular form. We conduct this study to investigated the efficacy in a beagle model.

\section{Methods}

\section{Urethroplasty using everted saphenous vein graft}

This experimental animal protocol was approved by the Animal Experimentation Ethics committee of Wannan Medical College in accordance with the ARRIVE guidelines. Twelve male beagle dogs weighing 8-10 kg were randomly divided into three groups: experimental group $(n=5)$, control group $(n=5)$ and normal group $(n=2)$.

After general anesthesia with 3\% pentobarbital, the beagles in experimental group and control group were placed on an operating table in supine position. The right thigh and genital organ were shaved and prepared with povidone-iodine solution. A 4-cm incision was carried out on the ventral skin of penis. A 3 $\mathrm{cm}$ segment of anterior urethra was removed between the external urethral orifice and bulbar urethra, in order to make a urethral defect(Fig.1A).

A 4-cm incision was carried out on the right thigh skin and a 3-cm saphenous vein graft was excised(Fig.1B). In experimental group, the vein grafts were everted (turned over to make the endothelial cells outside)(Fig.1C). A $8 \mathrm{~F}$ catheter was inserted into the urethra and was used as a stent(Fig.1D). Urethral defect was repaired by the everted saphenous vein graft in tubular fashion with 6-0 vicryl separated sutures(Fig.1E). In control group, the urethral defect was repaired by uneverted saphenous vein graft in the same manner. Nonabsorbable sutures were placed at the anastomosis margins for future mark. The subdermal layer in penis and the skin was closed(Fig.1F). In normal group, no surgical intervention was performed in animals. The procedures for all animals were performed under sterile conditions. All the procedures were performed by the same surgeon.

\section{Postoperative care}

An Elizabetan collar was placed around the neck of animal to prevent the catheter bite off. The catheter was left indwelling for two weeks. All animals in experimental group and control group received penicillin $100 \mathrm{U} / \mathrm{kg} /$ day intramuscularly for 14 days.

\section{Retrograde urethrography}

Retrograde urethrography was performed every month after operation. After general anesthesia, the animals were placed obliquely on an examination table. A Foley (6-F) catheter was inserted into the urethra until the balloon entered the urethral orifice. Inflate the balloon with $1 \mathrm{ml}$ sterilized water. X-ray images of urethra were obtained under the fluoroscopy after contrast agent was gently injected through the catheter. 


\section{Histological and immunohistochemical analysis}

Animals were euthanized by an overdose of $3 \%$ pentobarbital and air embolization 6 months after operation, the penises were obtained and the segments of the reconstructed urethra were extracted within the area outlined by the marking sutures. These segments were placed in $10 \%$ formalin for hematoxylineosin(HE) staining, Masson's trichrome(MT) staining, and immunohistochemical(IHC) staining using CD31 and uroplakin-II(UP-II).

\section{Results}

The surgical procedures were successfully performed on all beagles. There were no major intraoperative complications. During the 6 months follow-up period, one animal in experimental group and two in control group could not voided spontaneously. They underwent cystostomy with a suprapubic catheter. Urogenic cutaneous fistula in penis was detected in the other three animals in control group.

\section{Retrograde urethrography}

The retrograde urethrograms showed the urethral caliber of four beagles in experimental group were similar to that of normal beagles (Fig.2A,B). The contrast agent passed through the urethra freely without any signs of stricture or contrast leak. These indicate that the four beagles in experimental group could maintain wide urethral lumen 6 months postoperatively. The one beagle in experimental group could not voided spontaneously developed urethral stenosis. Ether significant urethral stricture or fistula were detected in all animals in control group (Fig.2C,D).

\section{Histological and IHC examination}

In experimental group, HE staining indicated the urethral lumen of the reconstructed segment was completely covered by endothelium(Fig.3E). There was no obvious difference with that of normal urethra(Fig.3A). MT staining showed highly organized collagen fibers and muscle tissue in subepithelium, but the muscle tissue seemed to be less than that of normal urethra(Fig.3B,F). The UP-II antibody is a special urothelium marker. IHC staining with UP-II antibody showed the expression of endothelium in the ureteral inner wall was positive(Fig.3G).

In control group, the reconstructed segment had a narrow urethral lumen and the endothelium was not intact(figure.3l). MT staining showed abundant collagen with strong blue staining, indicating severe fibrosis in subepithelium 6 months postoperatively(Fig.3J). IHC staining with UP-II antibody revealed a fragment of urothelium in the urethral inner wall(Fig.3K).

IHC staining using CD31 was performed to observe the neovascularization of implanted tissue. In experimental group, IHC staining with CD31 monoclonal antibody showed newly formed capillaries were dense after 6 months. But the density of vascular capillaries was low than that of normal urethra(Fig.3D,H). In control group, IHC staining using CD31 indicated sparse vascular capillaries(Fig.3L). 


\section{Discussion}

Various urethral conditions often require substitute urethral reconstruction including long-segment urethral strictures, traumatic defects, complicated hypospadias and previous failed urethroplasty[8]. Serveral substituted materials have been adopted for clinical urethroplasty: full-thickness skin graft, bladder mucosa, oral mucosa( buccal or lingual mucosa) and colonic mucosa[9-11]. Buccal mucosa and preputial skin graft remain the widely used tissues for urethral replacement, especially in cases of complex urethral reconstruction. However, there are risk of complications, such as graft necrosis, hair growth, stricture recurrence, and fistula formation[12,13]. In addition, harvesting buccal mucosa may also lead to possible morbidities such as intraoperative hemorrhage, submucosal scarring, pain, postoperative infection, and injury to salivary ducts[3][14]. Wood et al[15] reported the morbidity of buccal mucosa grafts harvested for urethroplasty in 57 patients. Of these patients, $68 \%$ had perioral numbness that persisted after 6 months in $26 \%$ of the patients, $83 \%$ developed postoperative pain, $67 \%$ initially had diffificulty with mouth opening that persisted after 6 months in $9 \%$ of the patients, and $2 \%$ had a mucous retention cyst. Dublin et al [6]reported that $57 \%$ patients had oral numbness after surgery (during the first 48 hours) that persisted for a year in $16 \%$ of patients and that $75 \%$ of patients complained of tightness of the mouth after surgery (during the first 48 hours) that persisted for nearly 2 years in $32 \%$ of cases. When urethral defect is long, donor tissue from oral cavity or prepuce may be insufficient. For these reasons, many attempts have been made to select alternative tissues that would serve as adequate urethral substitutes. Tissue engineering may be a promising option for the creation of artificial urethral tissues. But accompanied techniques are complex with multi-steps and the results were dissatisfactory in some preliminary clinical applications[16, 17]. Till now, tissue engineered urethra mainly at the stage of animal experiments so that it has not been widespread accepted in the clinic[18].

For anterior urethral strictures or hypospadias, a multi-stage urethroplasty in onlay fashion is generally recommended[4][19]. However, there is a lack of exploration of one-stage reconstruction using tubularized graft. In some cases, onlay grafts may not be suitable and tubularized urethroplasty is necessary[20, 21]. We previously performed urethroplasty in a rabbit model with saphenous vein patch as onlay graft and gained encouraging results[7]. Saphenous vein is a hollow tubular structure with a similar diameter of urethra. It has advantages of easy to be harvested and improved cosmetic outcomes, almost involves no injury to its donor site.

Tuffer performed urethral reconstruction using vein graft for the first time in 1910[13]. Subsequently, several animal trials have been reported using vein grafts for urethral reconstruction[22-24]. Hubner et al[24]and Foroutan et al[25]used everted vein grafts and their outcomes were improved. They thought their modified technique eliminated negative effects on urine stream from the valve inside the venous lumen. To the best of our knowledge, one of the key factors in reconstructing urethra is adequate blood supply and fast angiogenesis in implanted graft. Vascular density in the urethral cavity plays a central role in determining the fate of tissue repair[26, 27]. Insufficient blood supply inevitably results in shrinkage of grafts and fibrosis formation. In the study, the saphenous vein was everted and the vascular endothelium became outside. We hypothesize that the vascular endothelium could gain blood supply 
from periurethral muscles. We previously published our hypothesis that everted vein graft could survive without graft necrosis and slough off [7]. In this study, IHC staining with CD31 showed dense vascular capillaries in the experimental group. We demonstrate that everting the vein graft improved its ability of angiogenesis.

Adult male beagles were selected as our experimental model because the urinary tract in beagle resemble that of the human. Foroutan et al[25]performed histological study after euthanizing rabbits at days 7,10 , 14,22 , and 30 after operation, they found that gradual uroepithelialization occurred within one month. They demonstrated that the vein graft functioned as a guide for migration of uroepithelium. The histological examination in our previous experiment revealed similar progress[7]. The everted vein graft showed full integration to the neourethra. Urethral lumen was completely covered by regenerative uroepithelium 6 month postoperatively. Fistula formation, stenosis at the anastomosis were noted in the animals of control group. The experimental group showed improvement over the control group both in retrograde urethrography and histologic analysis. The MT staining showed that well-formed collagen and muscle fibers in experimental group. However, abundant collagen fiber, narrow urethral lumen and urothelium defect were found in control group. These results confirmed and supplemented our hypothesis. The rapid survival of the implant graft contribute to its function serving as a barrier of urine extravasation, facilitating urothelium cell migration and proliferation in the newly formed urethral tissue[28].

We create urethral defect models in a healthy urethra of normal animals, which cannot fully resemble the exact clinical situation of urethral stricture, which is characterized by the fibrotic urethra bed. Other limitations include a small sample size and a short follow-up time. The number of animals used in an experimental study is always a concern of the Ethics Committee, so the minimum number was used to answer the questions that were proposed. Further investigations with longer follow-up are necessary to assess its technical applicability and to translate this technology into clinic.

\section{Conclusion}

We first used the everted saphenous vein graft for tubularized urethral reconstruction to treat anterior urethral strictures and hypospadias. In our study, the experimental beagles had no voiding difficulty, no urethral fistula. And the animals had wide urethral lumen during the observation period. This study provides preclinical evidence and information needed to move toward clinical studies of this technique.

\section{List Of Abbreviations}

hematoxylin-eosin=HE; Masson's trichrome=MT; immunohistochemical=IHC; uroplakin-II=UP-II

\section{Declarations}

\section{Ethics approval and consent to participate}


The beagles in this research were acquired through the Animal Research Center of Wannan Medical College. Ethics approval was taken from Animal Experimentation Ethics committee of Wannan Medical College in accordance with the ARRIVE guidelines for reporting animal research.

\section{Consent for publication}

Not applicable

\section{Availability of data and materials}

All data generated or analysed during this study are included in this published article.

\section{Competing interests}

The authors report no conflicts of interest.

\section{Funding}

No funding or support received for this work.

\section{Authors' Contributions}

Protocol/project development: YX

Data collection or management: DL, ZS

Manuscript writing/editing $\triangle \mathrm{YX}, \mathrm{DL}$

All Authors read and approved the manuscript.

\section{Acknowledgements}

Not applicable

\section{References}

1. Barbagli G, Palminteri E, Lazzeri M, Guazzoni G. Anterior urethral strictures. BJU Int. 2003;92(5):497505.

2. Engel O, Fisch M. Urethral reconstruction after failed primary surgery.Urologe A. 2010;49(7):822-826.

3. Bhargava S, Chapple CR. Buccal mucosal urethroplasty: is it the new gold standard? BJU Int. 2004;93(9):1191-1193.

4. Lee OT, Durbin-Johnson B, Kurzrock EA. Predictors of secondary surgery after hypospadias repair: a population based analysis of 5,000 patients. J Urol. 2013;190(1):251-255. 
5. Sinha RJ, Singh V, Sankhwar SN, Dalela D. Donor site morbidity in oral mucosa graft urethroplasty: implications of tobacco consumption. BMC Urol. 2009;9:15.

6. Dublin N, Stewart LH. Oral complications after buccal mucosal graft harvest for urethroplasty. BJU Int. 2004;94(6):867-869.

7. Xu Y, Shen Z, Liu G, et al. Urethral Reconstruction Using Everted Saphenous Vein Graft in a Rabbit Model: One-Year Outcomes. Urol Int. 2017;99(1):110-117.

8. Peterson AC, Webster GD. Management of urethral stricture disease: developing options for surgical intervention. BJU Int. 2004;94(7):971-976.

9. Liu JS, Han J, Said M, et al. Long-term outcomes of urethroplasty with abdominal wall skin grafts. Urology. 2015;85(1):258-262.

10. O'Riordan A, Narahari R, Kumar V, Pickard R. Outcome of dorsal buccal graft urethroplasty for recurrent bulbar urethral strictures. BJU Int. 2008;102(9):1148-1151.

11. Xu Y-M, Qiao Y, Sa Y-L, Zhang J, Fu Q, Song L-J. Urethral reconstruction using colonic mucosa graft for complex strictures. J Urol. 2009;182(3):1040-1043.

12. Brannan W, Ochsner MG, Fuselier HA, Goodlet JS. Free full thickness skin graft urethroplasty for urethral stricture: experience with 66 patients. J Urol. 1976;115(6):677-680.

13. Vyas PR, Roth DR, Perlmutter AD. Experience with free grafts in urethral reconstruction. J Urol. 1987;137(3):471-474.

14. Rao AR, Shergill I, Thwaini A, Karim O, Motiwala H. Oral complications after buccal mucosal graft harvest for urethroplasty. BJU Int. 2005;95(4):679.

15. Wood DN, Allen SE, Andrich DE, Greenwell TJ, Mundy AR. The morbidity of buccal mucosal graft harvest for urethroplasty and the effect of nonclosure of the graft harvest site on postoperative pain. J Urol. 2004;172(2):580-583.

16. Mangera A, Chapple CR. Tissue engineering in urethral reconstruction--an update. Asian J Androl. 2013;15(1):89-92.

17. Dorin RP, Pohl HG, De Filippo RE, Yoo JJ, Atala A. Tubularized urethral replacement with unseeded matrices: what is the maximum distance for normal tissue regeneration? World J Urol. 2008;26(4):323-326.

18. Osman NI, Hillary C, Bullock AJ, MacNeil S, Chapple CR. Tissue engineered buccal mucosa for urethroplasty: progress and future directions. Adv Drug Deliv Rev. 2015;82-83:69-76.

19. Springer A, Krois W, Horcher E. Trends in hypospadias surgery: results of a worldwide survey. Eur Urol. 2011;60(6):1184-1189.

20. Macedo A, Srougi M. Onlay urethroplasty after sectioning of the urethral plate: early clinical experience with a new approach - the 'three-in-one' technique. BJU Int. 2004;93(7):1107-1109.

21. Macedo A, Liguori R, Ottoni SL, et al. Long-term results with a one-stage complex primary hypospadias repair strategy (the three-in-one technique). J Pediatr Urol. 2011;7(3):299-304. 
22. Kahveci R, Kahveci Z, Sirmali S, Ozcan M. Urethral reconstruction with autologous vein graft: an experimental study. Br J Plast Surg. 1995;48(7):500-503.

23. Kim BS, Kim HT, Kwon SY, et al. Nontransected ventral onlay-augmented urethroplasty using autologous saphenous vein graft in a rabbit model of urethral stricture. Urology;2014. 83(1):225-231.

24. Hübner W, Rurka I, Porpaczy P, Miko I. Autologous everted vein graft for repairing long-section urethral defects. Urol Res. 1991;19(2):131-134.

25. Foroutan HR, Khalili A, Geramizadeh B, Rasekhi AR, Tanideh N. Urethral reconstruction using autologous and everted vein graft: an experimental study. Pediatr Surg Int. 2006;22(3):259-262.

26. Jia $\mathrm{W}$, Tang $\mathrm{H}, \mathrm{Wu}$ J, et al.Urethral tissue regeneration using collagen scaffold modified with collagen binding VEGF in a beagle model. Biomaterials.2015;69:45-55.

27. Niu Y, Liu G, Chen C, et al. Urethral reconstruction using an amphiphilic tissue-engineered autologous polyurethane nanofiber scaffold with rapid vascularization function. Biomater Sci. 2020;8(8):21642174.

28. Orabi H, AbouShwareb T, Zhang Y, Yoo JJ, Atala A. Cell-seeded tubularized scaffolds for reconstruction of long urethral defects: a preclinical study. Eur Urol. 2013;63(3):531-538.

\section{Figures}
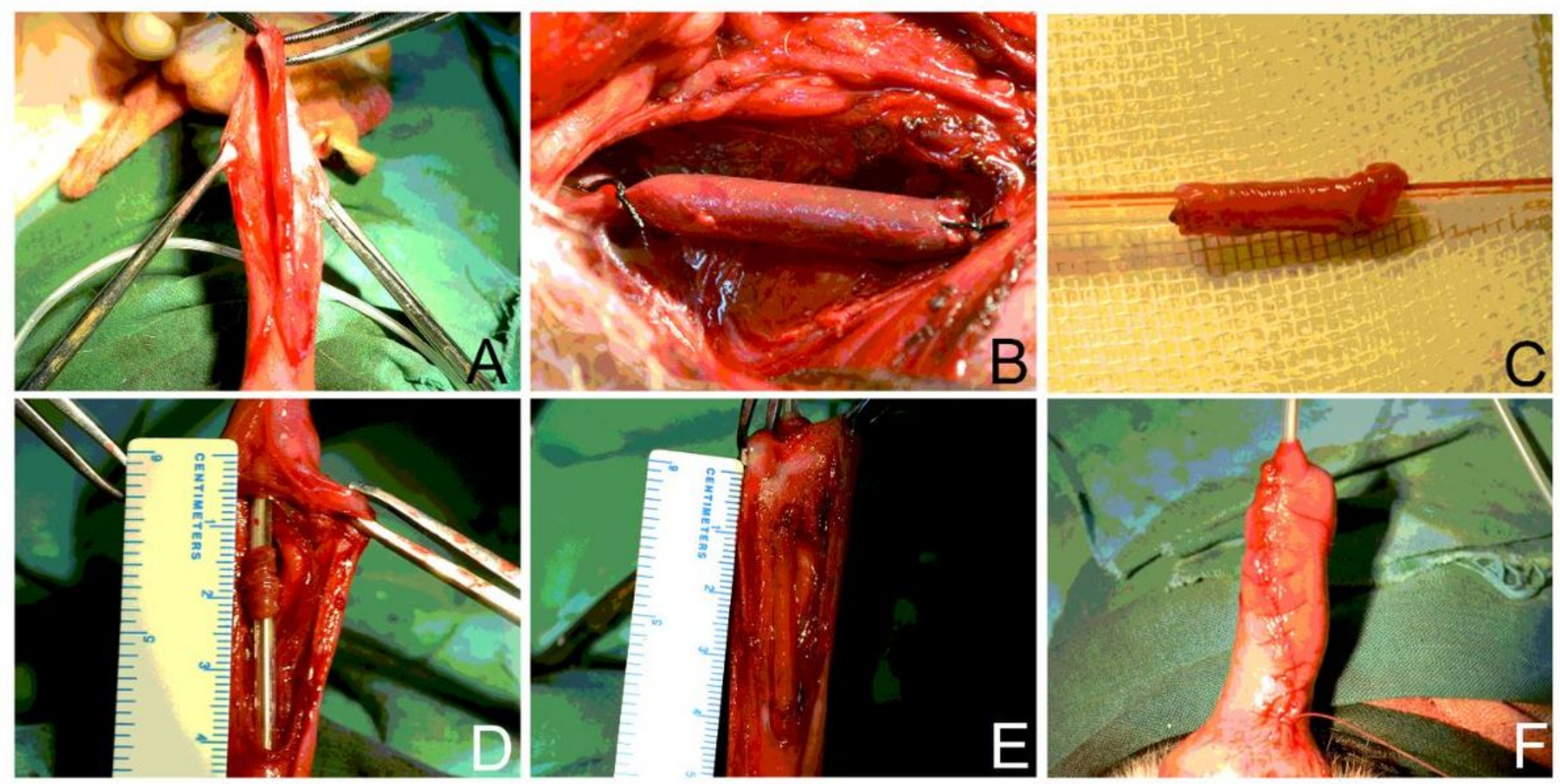

\section{Figure 1}

Anterior urethral reconstruction with saphenous vein graft. (A)A segment of anterior urethra was separated. (B)A 3-cm saphenous vein graft was harvested. (C) The saphenous vein graft was everted in 
experimental group. (D) The urethral catheter was inserted as a stent. (E)The urethral defect was repaired by saphenous vein graft in tubular fashion. (F)The subdermal layer in penis and the skin was closed.
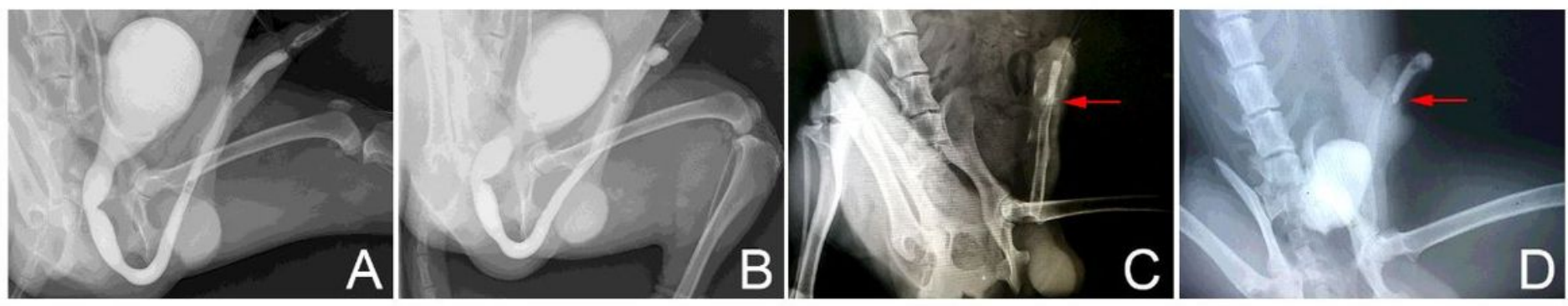

Figure 2

Retrograde urethrography. (A)Urethrogram in beagle in normal group. (B) Urethrogram in experimental group 6 months postoperatively. (C)Urethral fistula in control group(Red arrow point to the contrast leak). (D)Urethral stricture in control group(Red arrow point to the stricture).

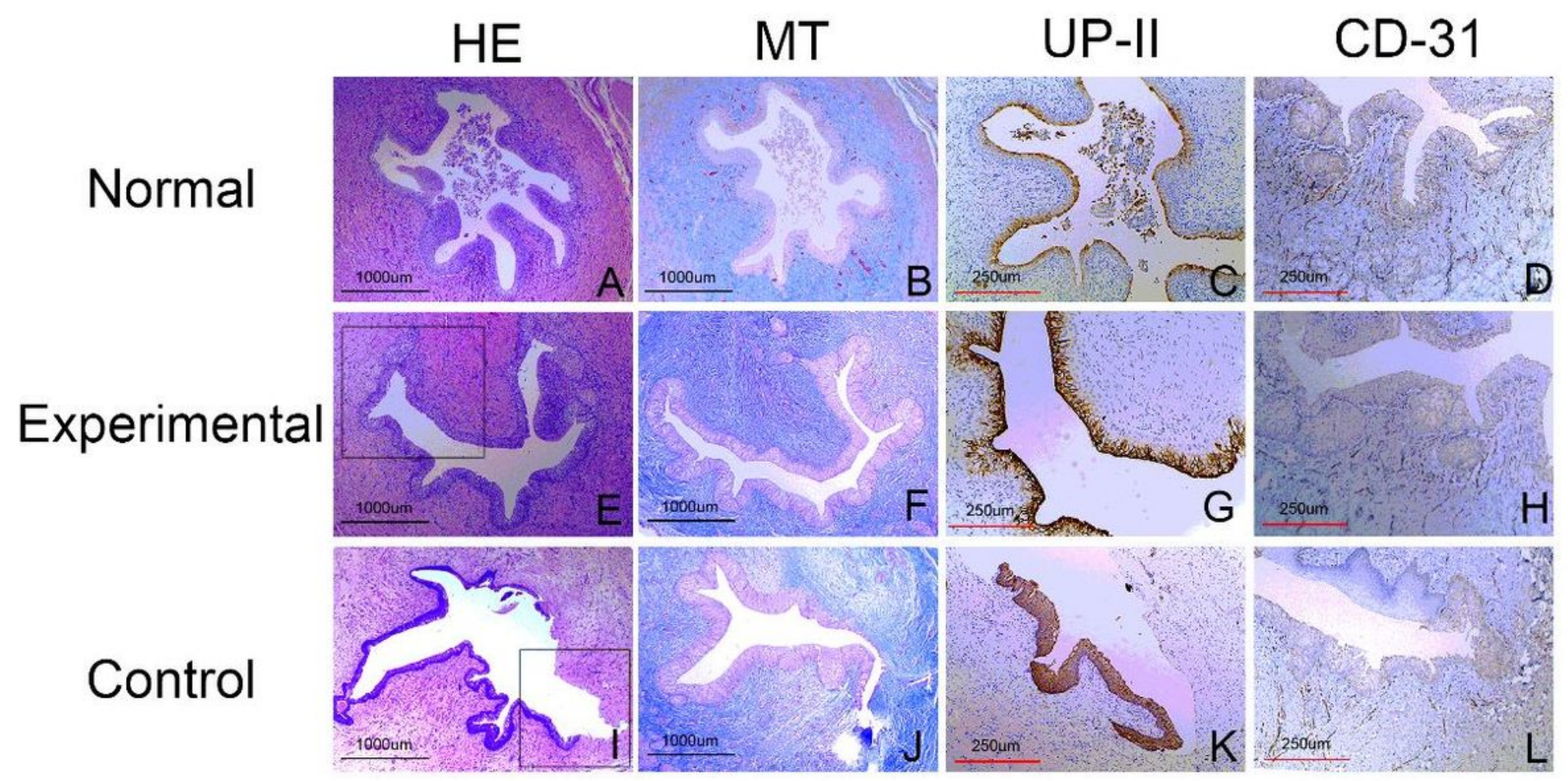

\section{Figure 3}

Histological and $\mathrm{IHC}$ analysis. (A,B,C,D) HE staining, MT staining, IHC staining with UP-II and CD31 of the reconstructed urethra in normal group. (E)Wide urethral lumen was completely covered by endothelium in experimental group. (F)MT staining showed highly organized collagen fibers and muscle tissue in subepithelium. (G)IHC staining with UP-II antibody showed expression of uroplakin in the endothelium. (H) IHC staining with the CD31 showed dense capillaries in subepithelium. (I)HE staining showed a 
narrow urethral lumen and urothelium defect in control group. (J)MT staining showed fibrosis with abundant collagen. (K)IHC staining with the UP-II revealed a fragment of urothelium. (L) Vascular capillaries was sparse in control group.

\section{Supplementary Files}

This is a list of supplementary files associated with this preprint. Click to download.

- TheARRIVEGuidelinesChecklist.pdf 